\title{
Tobacco Smoke Exposure, C-reactive Protein and Steroid Hormones Measured by Tandem Mass Spectrometry in Healthy Women
}

\section{Sarah H Chung ${ }^{1}$, Kepher H Makambi ${ }^{2}$ and Offie P Soldin ${ }^{3 *}$}

${ }^{1}$ Georgetown University School of Medicine, USA

${ }^{2}$ Departments of Biostatistics, Bioinformatics, and Biomathematics, Georgetown University, Lombardi Comprehensive Cancer Center, Washington, DC, USA

${ }^{3}$ Departments of Oncology, Medicine, Physiology and Biophysics, Obstetrics and Gynecology, Pharmacology and Medicine, Bioanalytical Core Laboratory, Georgetown University Medical Center; Lombardi Comprehensive Cancer Center, Washington, DC, USA

\begin{abstract}
Introduction: High sensitivity C-reactive protein (hsCRP) is a reliable biomarker of inflammation. Plasma CRP increases dramatically after severe trauma, bacterial infection and inflammation. The hsCRP accurately and sensitively measures basal levels of CRP, including in the areas of increased risk of developing myocardial infarction. Tobacco smoking has also been indicated to influence pregnancy outcomes and infertility.

Methods: We examined the associations between smoking, circulating hsCRP, and steroid hormone levels in healthy, non-pregnant women of reproductive age. Serum hsCRP concentrations were analyzed using immunoturbidimetry. Based on cotinine levels and self-questionnairres, the women were divided into three separate groups of smokers, nonsmokers and passive smokers (secondhand exposure). Steroids and cotinine levels were measured by isotope dilution tandem mass spectrometry.

Results: A significant, positive correlation was observed between hsCRP and cotinine levels indicating an association between cigarette smoking and inflammation. However, no association was found between hsCRP and cotinine levels in both the non-smoker and passive smoker group. Also, hsCRP levels were significantly associated with increased BMI scores.

Conclusions: Combined factors of increased smoke exposure and obesity were significantly correlated with increased hsCRP levels, suggesting that multiple conditions confer additive risk to an inflammatory state. To determine the role of inflammation in women's health, further studies are essential to determine the interacting relationship between hsCRP and sex hormone levels in women with disease.
\end{abstract}

Keywords: Biomarkers; Cotinine; Inflammation; Sex hormones; Gender; Secondhand/environmental exposure; Nicotine properties or derivatives; Public health

\section{Introduction}

Tobacco smoking and secondhand smoke exposure have been identified as agents that cause cardiovascular disease and precipitate cancer development. Smoke exposure, particularly for women, heavily influences pregnancy outcomes and is associated with infertility, preterm delivery, stillbirth, low birth weight, and sudden infant death syndrome.

The association between smoking and inflammation has been examined mainly in the context of cardiovascular disease. It was demonstrated that cigarette tobacco smoke causes direct vascular injury, subsequently leading to an immunological response [1]. These immunological responses involve the release of potent cytokines that stimulate hepatic synthesis of acute phase reactants, proteins that are thought to be anti-infective, anti-proliferative, and pro-coagulative.

In the mid-1990s, more sensitive methods for the measurement of CRP were introduced. These methods, referred to as hs-CRP, can accurately measure basal levels of CRP throughout the currently accepted cardiovascular risk assessment range (0.20-10.0 mg/L). The hs-CRP assays were used to assess outcomes in patients with unstable angina and showed that hs-CRP values in the upper tertile $(>3.0 \mathrm{mg} / \mathrm{L})$ were associated with increased risk of developing myocardial infarction $[1,2]$.

Much attention has been given to CRP as a potential biomarker for pathologies involving inflammation. As one of the first reactants released into plasma, its measurement has been considered a classic indicator for the acute phase response in inflammation. CRP is synthesized in the liver in response to interleukin (IL)-6, IL-1, and tumor necrosis factor (TNF) released from the inflamed tissue. It plays a key role in activating the complement system, inducing adhesion molecule expression, enhancing phagocytosis and macrophage and leukocyte activation and opsonization [1]. CRP levels may directly reflect the expression of an IL-6 polymorphic genotype [1]. Further, studies in monozygotic twins demonstrate that CRP is related to total and central obesity, blood pressure, and lipid profile independent of genetics [3].

CRP is easily and inexpensively measured compared to other inflammatory markers, making it an attractive candidate as a prognostic marker for inflammation. The high sensitivity CRP assay (hsCRP) can detect serum CRP concentrations in the range of 0 to 2 $\mathrm{mg} / \mathrm{L}$. Normal CRP levels are defined as $<10 \mathrm{mg} / \mathrm{L}$. Individuals with no disease exhibit CRP levels $\leq 1 \mathrm{mg} / \mathrm{L}$ [1]. CRP assays have been used to detect and monitor inflammation in conditions such as rheumatoid

*Corresponding author: Dr. Offie P. Soldin, Lombardi Comprehensive Cancer Center, NRB E207, Georgetown University Medical Center, 3970 Reservoir Road, N.W., Washington D.C. 20057, USA, Tel: 240-421-1010; Fax: 202-687-3060; Email: os35@georgetown.edu

Received February 05, 2014; Accepted October 31, 2014; Published November 04, 2014

Citation: Chung SH, Makambi KH, Soldin OP (2014) Tobacco Smoke Exposure, C-reactive Protein and Steroid Hormones Measured by Tandem Mass Spectrometry in Healthy Women. J Steroids Horm Sci 5: 147. doi:10.4172/2157-7536.1000147

Copyright: ( $) 2014$ Chung SH, et al. This is an open-access article distributed under the terms of the Creative Commons Attribution License, which permits unrestricted use, distribution, and reproduction in any medium, provided the original author and source are credited. 
arthritis, polymyalgia rheumatica, inflammatory bowel disease, and temporal arthritis. Physiologically, CRP levels are elevated during the third trimester of pregnancy, and in patients taking oral contraceptives, hormone replacement therapies, and during menopause. Elevated levels are also seen in obesity [1]. In children of 3-16 years of age, CRP levels were demonstrated to be higher in those of low socioeconomic status, attributable to recent illness or increased adiposity in these children [4].

Whereas high sensitivity CRP (hsCRP) assays have been used to detect and monitor inflammatory conditions such as rheumatoid arthritis, polymyalgia rheumatica, and inflammatory bowel disease, there still remains ambiguity regarding its use as a prognostic marker for other diseases that exhibit downstream inflammatory effects, such as atherosclerosis or obesity. Many contemporary studies suggest that increased adipose tissue induces a chronic and sub-acute inflammatory state. Whether or not these individuals exhibit higher hsCRP levels has not yet been shown. Additionally, hsCRP levels in the context of abnormal serum hormone levels have not been adequately explored. Data elucidating the role of inflammation in reproductive health can contribute to our understanding of how inflammation might be indicative of stress, infertility, and complications in pregnancy related to abnormal hormone levels.

While an inflammatory state has been shown to be associated with atherosclerosis and cardiovascular disease, studies examining the relationship between CRP and cardiovascular risk factors, such as obesity and smoking habits, remain uncertain. Our objective was to

\begin{tabular}{|c|c|c|c|c|c|}
\hline \multicolumn{6}{|c|}{ Study Demographic Data } \\
\hline & Total & $\begin{array}{l}\text { Active } \\
\text { smokers }\end{array}$ & $\begin{array}{l}\text { Passive } \\
\text { smokers }\end{array}$ & Non-smokers & \\
\hline Characteristic & $(n=293)$ & $(n=107)$ & $(n=86)$ & $(n=100)$ & $\mathrm{P}^{*}$ \\
\hline Age (years) & & & & & \multirow{5}{*}{0.69} \\
\hline$<28$ & 29.1 & 29.7 & 27.4 & 29.9 & \\
\hline $28-37$ & 23.4 & 27.7 & 17.8 & 23.7 & \\
\hline $38-47$ & 23.4 & 21.8 & 25 & 23.7 & \\
\hline$>47$ & 24.1 & 20.8 & 29.8 & 22.7 & \\
\hline \multicolumn{5}{|l|}{ Race/Ethnicity } & \multirow{5}{*}{$<0.01$} \\
\hline White & 46.5 & 51.5 & 34.1 & 51.5 & \\
\hline Black & 40.8 & 37.9 & 47.6 & 38.1 & \\
\hline Asian & 5.7 & 3.9 & 3.7 & 9.3 & \\
\hline Mixed/Other & 7 & 6.7 & 14.6 & 1.1 & \\
\hline \multicolumn{5}{|l|}{ Education level } & \multirow{6}{*}{0.32} \\
\hline$<$ high school & 3.7 & 2.8 & 5.9 & 3 & \\
\hline High School & 19.1 & 16.8 & 20 & 20.2 & \\
\hline Some college & 25.2 & 30.8 & 28.2 & 17.2 & \\
\hline College/technical & 32.2 & 31.8 & 24.7 & 39.4 & \\
\hline Professional & 19.8 & 17.8 & 21.2 & 20.2 & \\
\hline \multicolumn{5}{|l|}{ Household income } & \multirow{6}{*}{0.54} \\
\hline$<10,000$ & 30.7 & 32 & 31 & 29 & \\
\hline $10,000-30,000$ & 17.8 & 15.5 & 19 & 19 & \\
\hline $30,000-60,000$ & 33.2 & 35 & 35.7 & 29 & \\
\hline $60,000-90,000$ & 7 & 9.7 & 3.6 & 7 & \\
\hline$>90,000$ & 11.5 & 7.8 & 10.7 & 16 & \\
\hline \multicolumn{5}{|l|}{ Marital status } & \multirow{5}{*}{$<0.01$} \\
\hline Single & 73.7 & 81.3 & 69.8 & 72 & \\
\hline Married & 13 & 5.6 & 15.1 & 17 & \\
\hline Has partner & 4.3 & 7.5 & 3.5 & 0 & \\
\hline $\begin{array}{c}\text { Divorced/ separated/ } \\
\text { widowed }\end{array}$ & 9 & 5.6 & 11.6 & 11 & \\
\hline \multicolumn{3}{|c|}{${ }^{*} p$ values from chi-square test } & & & \\
\hline
\end{tabular}

Table 1: Distribution of Data on study characteristics by smoking status on the day the serum was obtained. examine the association between serum hsCRP concentrations and serum cotinine, a long-lived nicotine metabolite and classic indicator of smoking status. Additionally, we examined the associations between serum hsCRP levels, body mass index, and serum hormone levels, exploring a mechanism for the harmful effects of smoking on infertility, and complications in pregnancy through inflammation and certain steroid hormones [5].

\section{Methods}

\section{Subjects}

This is a cross-sectional, three-arm study on adult women. The study was approved by the Georgetown University Medical Center Institutional Review Board (IRB). Blood samples were collected from a total of 325 healthy, non-pregnant women. 293 women of reproductive age (18-45 years) completed all study requirements and are included. Exclusion criteria included illness, infection, immunological or endocrine disorders diagnosed within six months prior to blood draw, as well as surgery with general anesthesia within the prior three months. Women having used birth control pills, steroids, or immunosuppressive medications within the previous two months were excluded. Participants that were male, post-menopausal, or did not reside in Virginia (Arlington, Alexandria, Fairfax, Loudoun), Maryland, or the District of Columbia were also excluded from the study. A summary of subject demographics is presented in Table 1.

Study participants were identified as non-smokers, passive smokers (secondhand smoke (SHS) exposure [6] and as active smokers. The smoking status of these three groups was defined using: (1) serum cotinine levels (2) self-reported identification and (3) a combination of serum cotinine and self-reported identification. Cotinine, a stable metabolite of nicotine and with a half-life of 18-24 hours, was used as the biomarker for cigarette smoke exposure. Using these definitions the women were divided to the 3 smoking groups. First, subjects defined themselves as active smokers if they had smoked at least one cigarette in the previous five days. Passive smokers were defined as subjects who reported to have been regularly exposed to SHS (lived or worked with a smoker on a daily basis) within the previous six months. Non-smokers were subjects who had not smoked or been exposed to SHS within the previous six months. Smoking history was also collected using a validated smoking questionnaire described below. Serum cotinine analysis of the samples was conducted at the laboratory medicine Clinical Chemistry section at Children's Hospital Boston.

\section{Questionnaire}

All eligible study participants completed a structured, in-person interview and questionnaire conducted by a trained clinical research assistant dedicated to this study. The smoking questionnaire is based on the U.S. National Institutes of Health's National Cancer Institute Transdisciplinary Tobacco use Research centers (TTURC) questionnaire. Information collected included detailed medical history, reproductive health, tobacco smoke exposure history and use, dietary habits, and lifestyle. Each of the study participants also provided blood samples at the time corresponding to the follicular stage of her cycle. Subjects were instructed to make their appointments two to three days after the beginning of their menstrual flow. To minimize diurnal variation, blood samples were drawn in the morning hours between 7:00-11:30am. Blood was collected in two $10-\mathrm{mL}$ red top vaccutainer tubes without serum separators (for LC/MS/MS and immunoassay hormone analysis), processed, serum separated into multiple labeled (ID number only) aliquots and frozen at $-80^{\circ} \mathrm{C}$ until analysis. 
Citation: Chung SH, Makambi KH, Soldin OP (2014) Tobacco Smoke Exposure, C-reactive Protein and Steroid Hormones Measured by Tandem Mass Spectrometry in Healthy Women. J Steroids Horm Sci 5: 147. doi:10.4172/2157-7536.1000147

Page 3 of 6

\section{Steroid hormone analysis}

Steroid hormones analyses were conducted at the Bioanalytical Core Laboratory at Georgetown University Medical Center using isotope dilution LC/MS/MS API-5000 (Applied Biosystems, CA) equipped with an atmospheric pressure photoionization source and deuterium-labeled internal standards as described previously $[7,8]$. The detection limit was defined as the concentration two standard deviations above the response at zero doses. The sensitivity of each assay, within run (intra-assay) and between run (inter-assay) imprecision, as well as the coefficients of variation $(\% \mathrm{CV})$ for replicate quality control samples averaged for different concentrations of steroids were low and dependent on analyte concentrations (4-12\%). These are now standard and are conducted routinely at the Bioanalytical Core Laboratory at Georgetown University Medical Center. The measurement of estradiol

\begin{tabular}{|c|c|c|c|c|}
\hline \multicolumn{2}{|c|}{ Cotinine } & N= & Mean & Std. Deviation \\
\hline \multirow{2}{*}{$\begin{array}{l}\text { Cotinine } \\
(\mathrm{ng} / \mathrm{mL})\end{array}$} & Nonsmokers & 100 & 0.5 & 0 \\
\cline { 2 - 5 } & Passive smokers & 86 & 1.9 & 3.3 \\
\cline { 2 - 5 } & Smokers & 107 & 207.5 & 193.1 \\
\hline
\end{tabular}

Table 2: Serum Cotinine Concentrations for the three smokers groups. was precise and accurate down to $1 \mathrm{pg} / \mathrm{mL}$. Eleven steroid hormones were measured in one profile: four androgens: androstenedione (AE), dehydroepiandrosterone (DHEA), dehydroepiandrosterone sulfate (DHEAS) and testosterone ( $\mathrm{T}$, testosterone), the corticosteroids (aldosterone (A), 11-deoxycortisol (DOC), and cortisol (F), progestin 17- $\beta$-hydroxyprogesterone (17OHP), and estrogens17- $\beta$-estradiol $\left(\mathrm{E}_{2}\right)$ and estriol $\left(\mathrm{E}_{3}\right)$. Luteal hormone $(\mathrm{LH})$ and follicle-stimulating hormone (FSH) concentrations were measured using immunoassays to confirm that each subject was in the follicular phase of her menstrual cycle at the time of the blood draw (follicular phase: $\mathrm{LH}<20 \mu \mathrm{IU} / \mathrm{mL}$ and $\mathrm{FSH}$ $2.5-10.2 \mu \mathrm{IU} / \mathrm{mL}$ ).

\section{CRP analysis}

CRP concentrationswere determinedusinganimmunoturbidimetric high sensitivity CRP (hsCRP) assay on the Roche P Modular system (Roche Diagnostics, Indianapolis, IN), using reagents and calibrators from DiaSorin (Stillwater, MN). Analysis was conducted at the clinical and epidemiological research laboratory of Children's Hospital Boston Department of Laboratory Medicine. Serum CRP concentrations in healthy adults are usually lower than $10 \mathrm{mg} / \mathrm{L}$, slightly increasing with age. CRP levels are slightly higher in late pregnancy, viral infections

\begin{tabular}{|c|c|c|c|c|c|}
\hline Steroid Hormone & & $\mathbf{N}$ & Mean & Std. Deviation & Normal* range \\
\hline \multirow{3}{*}{ DHEAS (ug/dL) } & Nonsmokers & 100 & 144.7 & 52.5 & $10.0-237.0$ \\
\hline & Passive smokers & 86 & 145.5 & 75.4 & \\
\hline & Smokers & 107 & 137.7 & 62.7 & \\
\hline \multirow{3}{*}{ Aldosterone (ng/dL) } & Nonsmokers & 100 & 43.5 & 60.8 & $1.0-32.0$ \\
\hline & Passive smokers & 86 & 19.8 & 49.1 & \\
\hline & Smokers & 107 & 24.6 & 43.3 & \\
\hline \multirow{3}{*}{ Cortisol (ug/dL) } & Nonsmokers & 100 & 13.6 & 7.8 & $2.4-28.6$ \\
\hline & Passive smokers & 86 & 12 & 6.9 & \\
\hline & Smokers & 107 & 11 & 6.1 & \\
\hline \multirow{3}{*}{ 11-Deoxycortisol (ug/dL) } & Nonsmokers & 100 & 0.0198 & 0.0345 & $0.012-0.050$ \\
\hline & Passive smokers & 86 & 0.0129 & 0.0122 & \\
\hline & Smokers & 107 & 0.0141 & 0.0127 & \\
\hline \multirow{3}{*}{ Androstenedione (ng/dL) } & Nonsmokers & 100 & 79.3 & 47.1 & $50.0-250.0$ \\
\hline & Passive smokers & 86 & 69.4 & 44.3 & \\
\hline & Smokers & 107 & 63.7 & 35.9 & \\
\hline \multirow{3}{*}{ Testosterone (ng/dL) } & Nonsmokers & 100 & 29.6 & 17.7 & $16.0-50.0$ \\
\hline & Passive smokers & 86 & 37.7 & 64.3 & \\
\hline & Smokers & 107 & 32 & 54.3 & \\
\hline \multirow{3}{*}{$17-\mathrm{OH}$ progesterone $(\mathrm{ng} / \mathrm{dL})$} & Nonsmokers & 100 & 55 & 65 & $3.0-91.0$ \\
\hline & Passive smokers & 86 & 54.1 & 53.4 & \\
\hline & Smokers & 107 & 47.8 & 49.3 & \\
\hline \multirow{3}{*}{$\mathrm{DHEA}(\mathrm{ng} / \mathrm{dL})$} & Nonsmokers & 100 & 337.3 & 239.6 & $130.0-980.0$ \\
\hline & Passive smokers & 86 & 311 & 267.3 & \\
\hline & Smokers & 107 & 301.5 & 249.3 & \\
\hline \multirow{3}{*}{ Progesterone (ng/mL) } & Nonsmokers & 100 & 150.2 & 413.4 & $10.0-950.0$ \\
\hline & Passive smokers & 86 & 42.9 & 198.1 & \\
\hline & Smokers & 107 & 90.9 & 281.6 & \\
\hline \multirow{3}{*}{ Estrone $(\mathrm{pg} / \mathrm{mL})$} & Nonsmokers & 100 & 63.9 & 59.8 & $29.0-105.0$ \\
\hline & Passive smokers & 86 & 63 & 55 & \\
\hline & Smokers & 107 & 53 & 35.6 & \\
\hline \multirow{3}{*}{ Estradiol (pg/mL) } & Nonsmokers & 100 & 80.4 & 92.1 & $34.4-170.2$ \\
\hline & Passive smokers & 86 & 79.8 & 152.3 & \\
\hline & Smokers & 107 & 72.6 & 94.4 & \\
\hline \multirow{3}{*}{ SHBG (nmol/L) } & Nonsmokers & 100 & 64.3 & 40.2 & $9.3-75.2$ \\
\hline & Passive smokers & 86 & 62 & 40.3 & \\
\hline & Smokers & 107 & 68.6 & 46.8 & \\
\hline
\end{tabular}

* Normal reference intervals for females older than $18 \mathrm{y}$

Table 3: Serum Hormone Concentrations in the three smokers groups. 


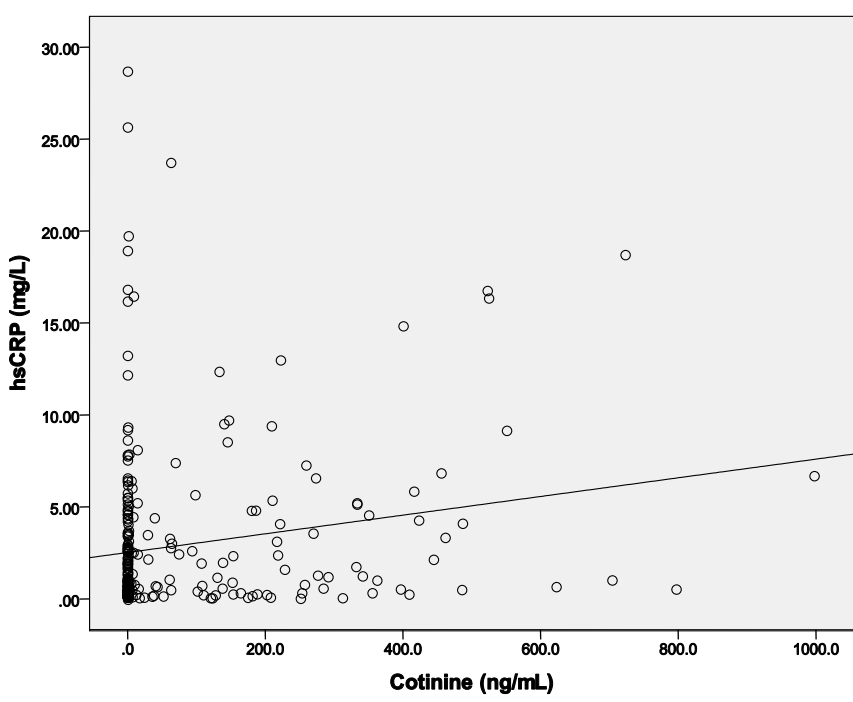

Figure 1: Scatter plot for the relationship between hsCRP and cotinine levels for all groups. Line of best fit indicates a positive correlation, $r^{2}=0.031$.

and mild inflammation $(10-40 \mathrm{mg} / \mathrm{L})$, active inflammation, bacterial infection $(40-200 \mathrm{mg} / \mathrm{L})$, and in severe burns and bacterial infections $(>200 \mathrm{mg} / \mathrm{L})[9]$.

\section{Statistical analysis}

Sample characteristics were presented as frequencies and percentages for categorical data. Scatter plots were constructed to obtain a visual impression of the association between two continuous variables. Chi-square tests and correlation coefficients were computed to assess the association between categorical variables and continuous variables, respectively. Analysis was conducted in SPSS version 19 for Windows. All statistical tests were two-sided and significance was judged at 0.05 levels.

\section{Results}

Serum cotinine and hormone concentrations are detailed in Tables 2 and 3.

Figure 1 illustrates a positive correlation between hsCRP levels and cotinine levels. There was a significant, positive correlation between hsCRP and cotinine levels in the active smokers group. Cotinine explained about $6 \%$ of the variance seen in hsCRP levels in this group. Moreover, in all groups, serum cotinine levels explain approximately $3 \%$ of the variance seen in hsCRP levels and indicated a significant predictor of hsCRP concentrations. When examining hsCRP levels within each of the three reclassified groups (non-, passive, and active smokers), however, no association was found between hsCRP and cotinine levels in both the non-smoker and passive smoker group.

hsCRP levels were significantly associated with increased BMI scores. BMI appeared to be a significant predictor of hsCRP after adjusting for age, explaining $15 \%$ of the variance seen in hsCRP levels. Stratified analysis by BMI indicated differences in mean hsCRP levels among normal $(\leq 25)$, overweight (25-29.99), and obese $(\geq 30)$ BMI categories. Significant differences were seen between the normal and obese groups and between the overweight and obese groups.

When classifying subjects into one of three BMI groups-normal (BMI < 25), overweight $(25-30)$, and obese $(>30)$ - there were no significant associations between hsCRP levels and a combination of the reclassified smoking status groups and the BMI groups. In addition, among individuals who were non-smokers there was a negative correlation between hsCRP levels and obesity (BMI > 30). A negative correlation was also observed between hsCRP levels and overweight status (BMI 25-30) in the reclassified passive smoker group. All other combinations yielded positive correlations.

One-way ANOVA indicates significant differences among the three smoking groups with combined risk factors of smoke exposure and obesity. Of note, mean hsCRP levels were significantly elevated in obese smokers compared to both non-obese non-smoker and non-obese smoker groups.

Correlation studies between hsCRP levels and cortisol, aldosterone, estrone, progesterone, and SHBG showed no significant associations between these serum hormones and hsCRP levels.

\section{Discussion}

Cigarette tobacco smoking is the second largest preventable public health cause of mortality and morbidity worldwide. Exposure to tobacco smoke is known to cause coronary heart disease, atherosclerosis and ischemic disease. The degree of this risk is proportional to cigarette tobacco smoke exposure, and it varies from individual to individual because of between-individual differences in genetic differences and other environmental exposures and behaviors. While the chemical properties of the tobacco smoke are relatively well characterized, the mechanisms by which smoking leads to disease and the genetic factors that determine susceptibility to these diseases are not well understood. Since smoking induces an inflammatory state, elevated CRP levels have been associated with smoking-related diseases. Levels of serum cotinine, a metabolite of nicotine, have been positively associated with CRP among smokers and non-smokers [10]. In adult smokers receiving 3.0-6.9 mg tar delivery per cigarette, hsCRP levels were greater than in non-smoking adults over a six-week period and proved to discriminate between the smoker and non-smoker groups with high sensitivity and specificity [6]. Individuals merely exposed to smoke also exhibit higher levels of CRP and increased risk for CVD-related death [11]. Lifetime secondhand smoke exposure appears to continually impact CRP levels.

Our results align well with recent studies exploring the relationship between elevated CRP levels and smoking-related diseases. We confirm the finding that tobacco smoke exposure is associated with increased levels of hsCRP. Cotinine, a nicotine metabolite with a half-life of 20 hours, has consistently been used as a valid indicator of cigarette smoke exposure among the tobacco researchers. A recent study by Benowitz et al. utilized cotinine to screen hospitalized patients and found that of individuals who denied smoking, $32 \%$ were found to have had significant smoke exposure [12]. Thus, cotinine is particularly useful in assessing secondhand smoke exposure and together with self-reported data, was used to represent smoking status in this study.

Blood concentrations of CRP are determined only by its rate of production since CRP clearance rate is constant. Following tissue insult, serum CRP levels will rise within 4-6 hours, increase exponentially within 24-48 hours and fall to normal levels only when tissue structure and function have been fully re-established [4]. In theory, this suggests that while CRP may be an adequate indicator of current injury, its presence in serum, when measured at a single point in time, cannot convey much regarding the chronicity of inflammation. However, recent studies have demonstrated that even after complete cessation of smoking, CRP levels remain high and do not fall to normal immediately, indicating that the tissue damage continues to provide an inflammatory 
stimulus [13]. In 152 smokers with CVD, hsCRP levels did not change with reduced smoking, nicotine-replacement therapy, and counseling six months later, further highlighting the prolonged effects smoking has on inflammatory response [14].

Previous studies have demonstrated that the number of cigarettes might be unrelated to the elevated levels of CRP seen in smokers (Summarized in Table 3). Ohsawa et al. found that CRP levels were elevated in all smokers, regardless of the number of cigarettes smoked per day, and that a longer smoking cessation period was associated with lower levels of CRP in past smokers [15]. Similarly, Asthana et al. studied leukocyte counts and CRP levels in active smokers and found positive correlations between smoking intensity and leukocyte counts but not with CRP levels [16]. This may suggest that smoking causes a substantial inflammatory response independent of nicotine dose. Further studies should focus on cigarette dosage to better elucidate these findings.

While a poor indicator of fat mass and distribution, BMI appeared to be positively associated with hsCRP levels in our study. This was true when examining each of the reclassified smoking groups individually. Previous studies have not only shown elevated levels of hsCRP in obesity but have also demonstrated sex differences in hsCRP levels and body fat [17]. The quantity and distribution of fat (truncal versus lower body) appear to influence CRP levels more strongly in women than in men. Levels of SHBG have been negatively associated with levels of CRP in postmenopausal women [18], and this relationship between SHBG and CRP has been demonstrated to be stronger in women with leaner body mass [19]. It is possible that various hormones may mediate the effects of obesity and inflammation in men and women. In an epidemiological study using fractional polynomials to identify the dose/response relationship between hsCRP levels and indicators of adiposity, it was found that the sex difference seen in hsCRP levels and adiposity disappears when adiposity is adjusted for leptin [20].

Importantly, combined factors of smoke exposure and obesity were shown in this study to be significantly correlated with increased hsCRP levels, suggesting that multiple conditions might confer additive risk to an inflammatory state. Matthews et al. found that women with sleep disordered breathing exhibited elevated levels of inflammatory and coagulation biomarkers (CRP, PAI, fibrinogen, VIIc) [21]. Increased adiposity, increased immunological responses to environmental toxins, i.e. tobacco smoke, and decreased sleep all may impose physiological stress to women. While the non-specific nature of CRP makes it difficult to identify the particular cause of its release into plasma, hsCRP levels may prove useful in obtaining a general picture of inflammation in women. It was unexpected to observe that age was not a significant predictor of hsCRP levels. The high incidence and development of chronic inflammatory conditions and atherosclerotic changes in older age was thought to potentially contribute to a positive correlation between age and hsCRP levels.

While this study found no significant correlations between hsCRP levels and serum hormone levels, there was a dose-dependent effect of smoke exposure associated with levels of serum estrone, estriol, estradiol, and 16-OHE1. Smoking appears to decrease levels of aromatase in granulosa cells, resulting in an anti-estrogenic effect in women. The lack of correlation between serum hormone levels and hsCRP may have been simply from the fact that the subjects examined were healthy women without illness, infection, immunological, or endocrine disorders. Future studies should examine hsCRP levels in women with abnormal hormone levels, or in a population of women having had a prior miscarriage, infertility, or adverse outcome in prior pregnancy, to further identify the inter-relationships between hsCRP and sex hormone levels.

Currently, there remains some debate regarding the use of hsCRP levels as a prognostic marker for disease. It is argued that hsCRP assays may prove less helpful for patients with different chronic co-morbidities due to the broad range of diseases that possess an inflammatory component [22].

Others argue that the use of hsCRP assays can greatly improve the identification of individuals at risk for CVD, particularly if they have unremarkable low-density lipoprotein (LDL) levels yet would still benefit from statin therapy [23]. The AFCAPS/TexCAPS study demonstrated that individuals with low LDL levels yet elevated hsCRP levels still exhibited high risk for CVD, and that lovastatin therapy proved more cost-effective in patients with high CRP levels $[24,25]$. Similarly, the Jupiter study exhibited that lovastatin therapy in individuals with normal lipid profiles and abnormal CRP levels ( $>$ $2 \mathrm{mg} / \mathrm{L}$ ) lowered their risk for CVD [24].

\section{Conclusions}

Inflammation as indicated by hsCRP levels is strongly associated with increased smoke exposure and increased BMI in healthy women of reproductive age. No significant correlations were found between hsCRP levels and serum hormone levels. These studies suggest that multiple factors, endogenous and environmental, are additive in promoting an inflammatory state. Further studies examining the inter-relationships between hsCRP and sex hormone levels in women with disease are essential in determining the role of inflammation in women's health.

\section{Declaration of Interests}

The authors declare no conflict of interests.

\section{Acknowledgement}

The authors would like to acknowledge Joshua Estrada, M.S. for his helpfu comments.

\section{Funding}

Dr. Offie Soldin is supported in part by NIH R01AG033867-01 and by FAMRI \#82533, the Flight Attendants Medical Research Institute.

\section{References}

1. Armani A, Becker RC (2005) The biology, utilization, and attenuation of C-reactive protein in cardiovascular disease: part II. Am Heart J 149: 977-983.

2. Armani A, Becker RC (2005) The biology, utilization, and attenuation of C-reactive protein in cardiovascular disease: part I. Am Heart J 149:971-976.

3. Greenfield JR, Samaras K, Jenkins AB, Kelly PJ, Spector TD et al. (2004) Obesity is an important determinant of baseline serum C-reactive protein concentration in monozygotic twins, independent of genetic influences. Circulation 109: 3022-3028.

4. Dowd JB, Zajacova A, Aiello AE (2010) Predictors of inflammation in U.S children aged 3-16 years. Am J Prev Med 39: 314-320.

5. Soldin OP, Guo T, Weiderpass E, Tractenberg RE, Hilakivi-Clarke L, et al (2005) Steroid hormone levels in pregnancy and 1 year postpartum using isotope dilution tandem mass spectrometry. Fertil Steril 84: 701-710.

6. Zedler BK, Kinser R, Oey J, Nelson B, Roethig HJ, et al. (2006) Biomarkers of exposure and potential harm in adult smokers of 3-7 mg tar yield (Federal Trade Commission) cigarettes and in adult non-smokers. Biomarkers 11: 201 220.

7. Guo T, Taylor RL, Singh RJ, Soldin SJ (2006) Simultaneous determination of 12 steroids by isotope dilution liquid chromatography-photospray ionization tandem mass spectrometry. Clin Chim Acta 372: 76-82.

8. Soldin OP, Makambi KH, Soldin SJ, O'Mara DM (2011) Steroid hormone levels associated with passive and active smoking. Steroids 76: 653-659. 
Citation: Chung SH, Makambi KH, Soldin OP (2014) Tobacco Smoke Exposure, C-reactive Protein and Steroid Hormones Measured by Tandem Mass Spectrometry in Healthy Women. J Steroids Horm Sci 5: 147. doi:10.4172/2157-7536.1000147

9. Clyne B, Olshaker JS (1999) The C-reactive protein. J Emerg Med 17: 10191025.

10. Jefferis BJ, Lowe GD, Welsh P, Rumley A, Lawlor DA, et al. (2010) Secondhand smoke (SHS) exposure is associated with circulating markers of inflammation and endothelial function in adult men and women. Atherosclerosis 208: 550556.

11. Hamer M, Chida $Y$ (2009) Associations of very high C-reactive protein concentration with psychosocial and cardiovascular risk factors in an ageing population. Atherosclerosis 206: 599-603.

12. Benowitz NL, Schultz KE, Haller CA, Wu AH, Dains KM et al., (2009) 3rd. Prevalence of smoking assessed biochemically in an urban public hospital: a rationale for routine cotinine screening. Am J Epidemiol 170: 885-891.

13. Hastie CE, Haw S, Pell JP (2008) Impact of smoking cessation and lifetime exposure on C-reactive protein. Nicotine Tob Res 10: 637-642.

14. Joseph AM, Hecht SS, Murphy SE, Lando H, Carmella SG, et al. (2008) Smoking reduction fails to improve clinical and biological markers of cardiac disease: a randomized controlled trial. Nicotine Tob Res 10: 471-481.

15. Ohsawa M, Okayama A, Nakamura M, Onoda T, Kato K, et al. (2005) CRP levels are elevated in smokers but unrelated to the number of cigarettes and are decreased by long-term smoking cessation in male smokers. Prev Med 41: 651-656.

16. Asthana A, Johnson HM, Piper ME, Fiore MC, Baker TB, et al. (2010) Effects of smoking intensity and cessation on inflammatory markers in a large cohort of active smokers. Am Heart J 160: 458-463.

17. Khera A, Vega GL, Das SR, Ayers C, McGuire DK, et al. (2009) Sex differences in the relationship between C-reactive protein and body fat. J Clin Endocrinol Metab 94: 3251-3258.
18. Maggio M, Ceda GP, Lauretani F, Bandinelli S, Corsi AM, et al. (2011) SHBG sex hormones, and inflammatory markers in older women. J Clin Endocrinol Metab 96: 1053-1059.

19. Joffe HV, Ridker PM, Manson JE, Cook NR, Buring JE et al., (2006) Sex hormone-binding globulin and serum testosterone are inversely associated with C-reactive protein levels in postmenopausal women at high risk for cardiovascular disease. Ann Epidemiol 16: 105-112.

20. Rossi IA, Bochud M, Bovet P, Paccaud F, Waeber G et al. (2012) Sex difference and the role of leptin in the association between high-sensitivity C-reactive protein and adiposity in two different populations. Eur J Epidemiol 27: 379-384.

21. Matthews KA, Zheng H, Kravitz HM, Sowers M, Bromberger JT, et al. (2010) Are inflammatory and coagulation biomarkers related to sleep characteristics in mid-life women?: Study of Women's Health across the Nation sleep study. Sleep 33: 1649-1655.

22. Tonstad S, Cowan JL (2009) C-reactive protein as a predictor of disease in smokers and former smokers: a review. Int J Clin Pract 63: 1634-1641.

23. Musunuru K, Kral BG, Blumenthal RS, Fuster V, Campbell CY, et al. (2008) The use of high-sensitivity assays for C-reactive protein in clinical practice. Nat Clin Pract Cardiovasc Med 5: 621-635.

24. Emerging Risk Factors Collaboration1, Kaptoge S, Di Angelantonio E, Lowe G Pepys MB, et al. (2010) C-reactive protein concentration and risk of coronary heart disease, stroke, and mortality: an individual participant meta-analysis. Lancet 375: 132-140.

25. Ridker PM (2007) C-reactive protein and the prediction of cardiovascular events among those at intermediate risk: moving an inflammatory hypothesis toward consensus. J Am Coll Cardiol 49: 2129-2138. 